Ágora Rev. Cient. 2018; 05(01):e3

\title{
Estrategias de adaptación de los padres en la crianza de niños con Síndrome de Down que acuden al Centro de Educación Básica Especial "Los Pinos" de San Juan de Lurigancho, 2017
}

\author{
Strategies for adaptation of parents in the upbringing of children with Down Syndrome attended at \\ Special Basic Education Center "Los Pinos" from San Juan de Lurigancho, 2017
}

Erika Stefani Melendez Cruz ${ }^{1, a}$, Ruth Elisabeth Quispe Damian ${ }^{1, a}$, Nadezhda Arias Romani ${ }^{1, b}$

\section{RESUMEN}

Objetivo: Describir las estrategias de adaptación de los padres en la crianza de niños con Síndrome de Down que acuden al Centro de Educación Básica Especial "Los Pinos" de San Juan de Lurigancho, 2017. Materiales y Métodos: El estudio fue observacional, de nivel descriptivo, de diseño no experimental. La muestra estuvo constituida por 38 padres de familia. Resultados: En relación a la estrategia del área afectiva, el 55.3\% manifestaron que se vincularon con otros padres que tienen hijos con síndrome de Down, mientras que el 18.4\% refirieron que buscaron apoyo espiritual, $13.2 \%$ padres se informaron sobre el síndrome de Down y con mismo porcentaje de $13.2 \%$ corresponde a los padres que buscaron apoyo emocional. En las estrategias de área de la salud, 81.6\% de padres afirman que sus hijos actualmente reciben terapias, y el $18.4 \%$ que no recibe terapias. Referente a los niños con SD que reciben terapias el $71 \%$ opta por la terapia de lenguaje, el $29 \%$ por la terapia de lenguaje junto con la psicomotriz. En cuanto a la estrategia del área social, el $76.3 \%$ de padres manifestaron que sí tuvieron el apoyo de su familia, y el $23.7 \%$ refieren que no tuvieron apoyo familiar. Dentro del grupo de padres que tuvieron apoyo familiar el $44.8 \%$ menciono que tuvieron aceptación de su persona y de su hijo, y el 13.8\% refieren que los familiares ayudaron a cuidar al niño. Y n la estrategia del área educativa, en el inicio de la vida escolar del niño con SD se evidencia que el 73.7\% de los padres optaron que sus hijos inicien su vida escolar en un colegio de educación especial, y en menor porcentaje $26,3 \%$ corresponde a los padres que optaron por el colegio de educación regular. Conclusión: Los niños se integran en las actividades ordinarias dentro del entorno social y familiar. Se ha de resaltar la conducta favorable frente a las actividades del entorno. Inicialmente los padres muestran cierto grado de preocupación debido a la dificultad para asimilar la situación respecto al estado de sus hijos.

Palabras claves: Síndrome de Down, crianza, estrategias.

\begin{abstract}
Objectives: Describe the strategies of adaptation of parents in the upbringing of children with Down syndrome that go to the Special Basic Education Center "Los Pinos" of San Juan de Lurigancho, 2017. Materials and Methods: The study was observational, descriptive level, non-experimental design. The sample consisted of 38 parents. Results: In relation to the strategy of the affective area, 55.3\% stated that they were linked with other parents who have children with Down syndrome, while $18.4 \%$ reported that they sought spiritual support, $13.2 \%$ parents were informed about Down syndrome and with the same percentage of $13.2 \%$ corresponds to parents who sought emotional support. In health area strategies, $81.6 \%$ of parents affirm that their children currently receive therapies, and $18.4 \%$ do not receive therapies. Regarding children with SD who receive therapies $71 \%$ choose language therapy, $29 \%$ for language therapy along with the psychomotor. Regarding the strategy of the social area, $76.3 \%$ of parents stated that they did have the support of their family, and $23.7 \%$ report that they did not have family support. Within the group of parents who had family support, $44.8 \%$ mentioned that they had accepted their person and their child, and $13.8 \%$ said that family members helped take care of the child. And in the strategy of the educational area, in the beginning of the school life of the child with SD it is evident that $73.7 \%$ of the parents opted for their children to start their school life in a special education school, and in a lower percentage $26.3 \%$ corresponds to parents who opted for the regular education college. Conclusion: Children are integrated into ordinary activities within the social and family environment. We must highlight the favorable behavior against the surrounding activities. Initially the parents show some degree of concern due to the difficulty to assimilate the situation regarding the status of their children.
\end{abstract}

Keywords: Down syndrome, parenting, strategies.

${ }^{1}$ Universidad María Auxiliadora.

${ }^{\mathrm{a} A u t o r}$

${ }^{\mathrm{b}} \mathrm{Co}$-autor 


\section{INTRODUCCIÓN}

El Síndrome de Down (SD) es un acontecimiento genético causado por tener una copia adicional del cromosoma 21. Las células humanas contienen 23 pares de cromosomas. La trisomía es un error que hace que, en uno de los pares en vez de haber dos cromosomas hay tres. En este caso las células contienen 47 cromosomas. Diferentes estudios parecen apuntar a que el cromosoma extra es de origen materno, es decir, el cromosoma de más estaría presente en el óvulo de la madre. La causa de la trisomía sería la ausencia de división de los cromosomas, error que puede ocurrir tanto en la primera como en la segunda fase de la meiosis. La probabilidad está asociada con la edad de la madre, incrementándose progresivamente a partir de los 35 años de ésta (1).

Entre las anomalías relacionadas al síndrome de Down están los problemas oculares; del $60 \%$ al $80 \%$ tienen un déficit auditivo y del $40 \%$ al $45 \%$ padecen alguna enfermedad cardíaca congénita. Los niños con síndrome de Down presentan comúnmente anomalías intestinales con mayor frecuencia que el resto de los niños, también padecen enfermedades cardíacas graves que suelen mostrar un retraso en el desarrollo (2).

Una gestante joven tiene un riesgo de 1 en 2000 de tener un niño con SD, mientras una gestante de 40 años tiene un riesgo de 1 en 100 debido a un aumento de no disyunción cromosómica durante la meiosis materna y a una menor selectividad uterina, sin embargo, a pesar de que la probabilidad de la gestante joven es menor, la frecuencia global de niños nacidos con $\mathrm{SD}$ es mayor en las parejas jóvenes porque estas madres se encuentran en plena edad fértil (3).

Se calcula que cada año 276.000 recién nacido fallecen durante las primeras cuatro semanas de vida en el mundo debido a anomalías congénitas. Las anomalías congénitas pueden ocasionar discapacidades crónicas con gran impacto en los afectados, sus familias, los sistemas de salud y la sociedad. Los trastornos congénitos graves más frecuentes son las malformaciones cardíacas, los defectos del tubo neural y el síndrome de Down (4).

Las mejoras aplicadas a la atención médica de las personas con Síndrome de Down han hecho aumentar la esperanza de vida y disminuir los niveles de mortalidad, aun así, siguen teniendo importantes problemas de salud, especialmente en sus primeros años de vida. Se identificó a los primeros 3 años como el principal periodo de hospitalización de niños con síndrome de Down. Entre los bebés de menos de un año que mueren durante su primer año, la mayoría de los estudios distinguen entre la mortalidad neonatal (muerte antes de los 28 días) y post-neonatal (entre 28 días y un año). En los bebés con síndrome de Down ocurrían proporcionalmente más muertes en el periodo post-neonatal (5).

El Estudio Colaborativo Latinoamericano de Malformaciones Congénitas (ECLAMC) en el año 2011, indica que Chile es el país de América Latina que cuenta con más casos de niños con Síndrome de Down, habiendo aumentado de 1,8\% por cada 1.000 nacimientos a mediados de los años 80 a $2,4 \%$ en esta década. Una de las explicaciones podría estar en el retraso de la edad materna por parte de las chilenas.

Sólo el 20\% de los casos se detecta durante el embarazo, por eso uno de los temas abordados es el diagnóstico prenatal a través de ecografías y marcadores químicos, el conocer tempranamente si el niño tiene esta condición distinta permite que sus padres puedan prepararse mejor para la llegada del bebé y como lo recibirán (6).

El Registro Nacional de la Persona con Discapacidad a cargo del Consejo Nacional para la Integración de las Personas con Discapacidad (CONADIS) al 2015 tiene inscritas un total de 141 731 personas, de las cuales 8800 tienen Síndrome de Down, representando el $6.21 \%$ del total de los registros.

El $44.7 \%$ son mujeres y el $55.3 \%$ son hombres, lo que en valores absolutos equivale a 3937 mujeres y 4863 hombres con Síndrome de Down inscritos en el Registro Nacional de la Persona con Discapacidad.

La mayor parte de las personas con Síndrome de Down inscritas en el Registro del CONADIS del año 2015 se ubica en la región Lima Metropolitana con 3766 registros $(42.80 \%)$, seguido de la región Piura con 498 (5.66\%) y La Libertad con 457 (5.19\%) personas inscritas, los menores porcentajes de $0.67 \%$ y menos corresponden a Huancavelica, Pasco, Moquegua, Ucayali y Madre de Dios (7).

En el distrito de San Juan de Lurigancho se estima que los menores de 17 años constituyen el $30 \%$ de la población y que son aproximadamente 340000 niños y adolescentes, de estos unos 50000 mil tendrán algún trastorno o discapacidad entre ellos el Síndrome de Down. No se encuentran datos específicos referidos a niños con Síndrome de Down, pero contamos con instituciones que brindan el servicio de atención y educación al niño con síndrome de Down, San Juan de Lurigancho cuenta con cuatro Programas de Intervención Temprana (PRITE) que atienden ambulatoriamente a niños menores de 3 años, y 7 Centros de Educación Básica Especial (CEBE) 
que atienden a niños a partir de 3 años (8). Los Pinos es un CEBE que cuenta con 25 años de funcionamiento y 19 secciones distribuidas entre turno mañana y tarde, donde encontramos niños con diferentes discapacidades, en la actualidad tenemos la cantidad de 38 niños con SD.

Esta realidad evidencia un problema de salud pública por su creciente incremento de niños con SD. Cuando nace el niño con Síndrome de Down, la familia con frecuencia no ofrece oportunidades suficientes para desarrollar aquellas habilidades y competencias personales y sociales, no siendo capaces de relacionarse y de actuar satisfactoriamente en el ámbito social. El soporte principal de los niños que acuden al CEBE Los Pinos lo constituyen los padres, al conversar con ellos manifiestan que aún no se han adaptado a la crianza de sus hijos, y expresan sentimientos de tristezas preocupación y frustración, otros no brindaron las facilidades para las entrevistas incluso refiriendo que sus hijos no tenían el síndrome de Down.

\section{MATERIALES Y MÉTODOS}

Por el tipo de intervención del investigador es de tipo observacional. Por el número de mediciones de las variables el estudio es transversal. Por el tiempo de recolección de datos es prospectivo. La población de estudio estuvo compuesta por los 38 padres de los 38 niños con Síndrome de Down, del Centro de Educación Básica Especial Los Pinos del distrito San Juan de Lurigancho 2017. Se aplicó un cuestionario, elaborado en la investigación, a los padres. En los resultados se aplicó análisis descriptivo.

\section{RESULTADOS}

Se presentan los resultados del estudio de acuerdo a los objetivos formulados, los datos son considerados en las tablas respectivas de acuerdo a cada uno de los objetivos.

La Tabla $\mathrm{N}^{\circ} 1$ muestra que estrategia utilizaron los padres de hijos con síndrome de Down para poderse adaptar afectivamente, 21 padres manifestaron que se vincularon con otros padres que tienen hijos con síndrome de Down (55.3\%), mientras que 7 padres refirieron que buscaron apoyo espiritual (18.4\%), 5 padres se informaron sobre el síndrome de Down (13.2\%) y con igual porcentaje $(13.2 \%)$ corresponde a 5 padres que buscaron apoyo emocional.
Tabla $N^{\circ}$ 1: Estrategias de adaptación en el área afectiva de los padres en la crianza de niños

\begin{tabular}{ccc}
\hline & Frecuencia & Porcentaje \\
\hline $\begin{array}{c}\text { Apoyo } \\
\text { emocional }\end{array}$ & 5 & $13.2 \%$ \\
Apoyo espiritual & 7 & $18.4 \%$ \\
$\begin{array}{c}\text { Informarse del } \\
\text { síndrome de } \\
\text { Down }\end{array}$ & 5 & $13.2 \%$ \\
$\begin{array}{c}\text { Vinculación con } \\
\text { otros padres }\end{array}$ & 21 & $55.3 \%$ \\
\hline Total & $\mathbf{3 8}$ & $\mathbf{1 0 0 \%}$
\end{tabular}

En la Tabla $\mathrm{N}^{\circ} 02$ podemos observar que 31 padres afirman que su hijo recibe terapias siendo el $81.6 \%$, y con menor porcentaje de $18.4 \%$ se encuentran los 7 padres que manifestaron que actualmente su hijo no recibe ningún tipo de terapia. De los padres que refirieron que su hijo recibe terapias el $71 \%$ opta por la terapia de lenguaje y el $29 \%$ por la terapia de lenguaje junto con terapia psicomotriz.

Tabla $N^{\circ}$ 2: Estrategias de adaptación que utilizan los padres en el área de la salud para la crianza de niños con Síndrome de Down.

\begin{tabular}{|c|c|c|c|c|c|}
\hline & $\mathrm{N}^{\circ}$ & Porcentaje & $\begin{array}{l}\text { Tipo de } \\
\text { terapias }\end{array}$ & $\mathrm{N}^{\circ}$ & Porcentaje \\
\hline \multirow[b]{2}{*}{$\mathrm{Si}$} & \multirow[b]{2}{*}{31} & \multirow[b]{2}{*}{81.6} & $\begin{array}{l}\text { Terapia de } \\
\text { lenguaje }\end{array}$ & 22 & $71 \%$ \\
\hline & & & $\begin{array}{c}\text { Terapia de } \\
\text { lenguaje y } \\
\text { terapia } \\
\text { psicomotriz }\end{array}$ & 9 & $29 \%$ \\
\hline No & 7 & 18.4 & & & \\
\hline Total & 38 & 100.0 & & 31 & $100 \%$ \\
\hline
\end{tabular}

En la Tabla No 3 se observa que $76.3 \%$ padres manifestaron que sí tuvieron el apoyo de su familia, y el $23.7 \%$ refieren que no tuvieron apoyo familiar. Dentro del grupo de padres que tuvieron apoyo familiar el $44.8 \%$ menciono que tuvieron aceptación de su persona y de su hijo, el 41,4\% que la familia acepta al niño, y con menor porcentaje $13.8 \%$ los padres refieren que los familiares ayudaron a cuidar al niño.

En la Tabla $\mathrm{N}^{\circ} 4$ se evidencia que el $73.7 \%$ de los padres de niños con síndrome de Down optaron que sus hijos inicien su vida escolar en un colegio de educación especial, y en menor porcentaje $26,3 \%$ corresponde a los padres que optaron por el colegio de educación regular para el inicio escolar de sus hijos. 
INVESTIGACIÓN ORIGINAL ORIGINAL RESEARCH
Estrategias de adaptación de los padres en la crianza de niños con Síndorme de Down que acuden al Centro de Educación Básica Especial "Los Pinos"
Tabla $\mathbf{N}^{\circ}$ 3: Estrategias de adaptación en el área social de los padres en la crianza de niños con Síndrome de Down.

\begin{tabular}{|c|c|c|c|c|c|}
\hline & $\mathbf{N}^{\circ}$ & Porcentaje & $\begin{array}{c}\text { Apoyo } \\
\text { familiar }\end{array}$ & $\mathbf{N}^{\circ}$ & Porcentaje \\
\hline \multirow{3}{*}{$\mathrm{Si}$} & \multirow{3}{*}{29} & \multirow{3}{*}{76.3} & $\begin{array}{l}\text { Cuidaron } \\
\text { al niño }\end{array}$ & 4 & 13.8 \\
\hline & & & $\begin{array}{c}\text { Aceptación } \\
\text { del niño }\end{array}$ & 12 & 41.4 \\
\hline & & & $\begin{array}{c}\text { Aceptación } \\
\text { de niño y } \\
\text { madre }\end{array}$ & 13 & 44.8 \\
\hline No & 9 & 23.7 & & & \\
\hline Total & 38 & 100.0 & & 29 & 100.0 \\
\hline
\end{tabular}

Tabla $N^{\circ}$ 4: Estrategias de adaptación en el área educativa de los padres en la crianza de niños con Síndrome de Down

\begin{tabular}{lcc}
\hline & $\mathbf{N}^{\circ}$ & Porcentaje \\
\hline Colegio de educación & 28 & 73,7 \\
básica especial & & \\
Colegio de educación regular & 10 & 26,3 \\
\hline \multicolumn{1}{c}{ Total } & 38 & 100,0 \\
\hline
\end{tabular}

\section{DISCUSIÓN}

En el presente estudio se identificaron las estrategias que fueron consideradas de manera frecuente por los padres de familia en la crianza de los niños con síndrome de Down. Estas estrategias de acuerdo al factor afectivo evidencian que los padres tras la primera impresión por el nacimiento de sus hijos con Síndrome de Down presentan sentimientos de tristeza y preocupación en la mayoría de los casos, pero a pesar de ello los padres buscan diferentes maneras de afrontar, como la búsqueda de apoyo emocional, informarse del síndrome de Down, apoyo espiritual, y vincularse con otros padres de niños con síndrome de Down, su comportamiento involucra signos de afectividad durante la crianza de sus hijos es por eso que la mayoría opta por buscar ayuda en otros padres de niños con Síndrome de Down y compartir sus experiencias. El estudio realizado por Acevedo B, Marriaga A, Zuliani Arango (13) se señala que la experiencia de crianza comienza, en la mayoría de las madres con una gran tristeza en el encuentro con su hijo con Síndrome de Down, dadas las preconcepciones sociales, luego aparece la alegría y una crianza amorosa como una posibilidad de interrelación entre madres e hijos. Además, Arias D, García A. (10) en su estudio se identificó que inicialmente para las madres es difícil asumir la noticia, pero a medida que van asumiendo el rol de madres que tienen que desempeñar, ellas adaptan y modifican conductas en pro del bienestar del hijo con síndrome de Down, sacrificando diferentes aspectos de su vida tanto a nivel personal, familiar y social para el desarrollo adecuado de su hijo.

De acuerdo al factor de salud, la mayoría de padres decidió buscar ayuda psicológica $65.8 \%$, y el $34.2 \%$ padres refiere no tomar la decisión de buscar ayuda psicológica. Siendo esta una estrategia fundamental para una buena afrontación al problema y consecuente adaptación a la crianza. Estos resultados concuerdan con el estudio realizado por Cruz V. (17) en donde señala que si existe una buena relación entre el bienestar psicológico y las actitudes que toman las madres para una mejor crianza.

De acuerdo al factor social, para los padres de niños con Síndrome de Down es muy importante el apoyo que reciben de su familia, en los resultados se obtuvo que $76,3 \%$ de los casos recibieron apoyo de parte de la familia, y $23.7 \%$ no tuvo apoyo familiar en primera instancia; los padres que sí tuvieron apoyo familiar lo recibieron de diferentes maneras como ayudando a cuidar al niño con síndrome de Down 4 (13.8\%) casos, la familia acepto al niño $12(41.4 \%)$ casos, y aceptaron al niño y madre conjuntamente evidenciándose con mayor porcentaje 13 (44.8\%). Estos resultados concuerdan con el estudio realizado por Chumo M, Zambrano M. (11) en donde se señala que los niños presentan un desarrollo afectivo fortalecido debido a las atenciones y muestras de cariño que le brinda la familia, fijando en ellos un tipo de apego seguro permitiéndoles además un mejor desarrollo integral. El estudio de Gomez T, Helguero I, (18) demuestra que la aplicación de programas para socialización de los niños con síndrome de Down responde de manera positiva para mejorar las habilidades sociales con una ganancia de $50 \%$ considerándose nivel alto, también hay nivel alto en la dimensión alternativa de agresividad. Además, la teórica Callista Roy menciona que en relación a su teoría la familia también es un receptor de cuidados, que también tiene una conducta de adaptación. El desarrollo que la familia hace es por activación de un proceso de aprendizaje.

De acuerdo al factor educacional, según resultados de nuestro estudio los padres optaron por que sus hijos inicien su vida escolar, el 28 $(73.7 \%)$ de niños iniciaron su vida escolar en un colegio de educación especial, y el 10 (26.3\%) de niños con Síndrome de Down lo hicieron en un colegio de educción regular; la mayoría de padres opto por el colegio de educación especial para 
evitar la discriminación. El estudio realizado por Tinoco W. (9) se relaciona ya que señala que los niños con síndrome de Down no serán educados de la misma manera que se hace con otros niños, sino de manera adecuada a la edad mental que el niño posea.

\section{CONCLUSIONES}

Las estrategias de adaptación en el área afectiva de los padres en la crianza de niños con Síndrome de Down ayudaron favorablemente en la mayoría de los casos. Los padres para poder adaptarse afectivamente buscan ayuda en otros padres que tienen el mismo caso de tener un hijo con síndrome de Down, ya que ellos pudieron dar una mejor consejería de cómo manejar la crianza de su hijo hasta llegar a mostrar aceptación y no rechazo por parte de ellos. Las estrategias de adaptación que utilizan los padres en el área de la salud para la crianza de niños con Síndrome de Down ayudaron favorablemente en la mayoría de los casos, con la realización de terapias de lenguaje y psicomotriz para sus hijos, y así llevar un mejor desarrollo de sus capacidades. Las estrategias de adaptación en el área social de los padres en la crianza de niños con síndrome de Down, fueron aplicadas favorablemente. Cabe mencionar que en la mayoría de los casos los padres recibieron el apoyo familiar que necesitaban integrando a los niños dentro del entorno familiar. Las estrategias de adaptación en el área educativa de los padres en la crianza de niños con Síndrome de Down ayudaron favorablemente en la mayoría de los casos. Desarrollar su vida escolar reforzará el proceso de aprendizaje y desarrollo en los niños, refortaleciendo su autonomía.

\section{REFERENCIAS BIBLIOGRÁFICAS}

1. Madrigal Muñoz Ana. El síndrome de Down. [Internet]. 2012 [citado el 5 de Mar. del 2012. Disponible desde: http://sid.usal.es/idocs/F8/FDO10413/infor me_down.pdf.

2. Organización de las Naciones Unidas [Internet]. Nueva York: Asamblea general [citado el 25 de feb. De 2017]. Disponible desde: http://www.un.org/spanish/News/stor y.asp?NewsID=31951\#.WjkfuYThDI U

3. Michel E. Weijerman, A. Marceline Furth, Antonie Vonk Noordegraaf, Jacobus P. Wouwe, Chantal J.M. Broers, Reinoud Gemke. Prevalencia, características neonatales y mortalidad durante el primer año del síndrome de Down: Un estudio nacional. Síndrome de Down 2010; 152: 15 19.

4. Organización Mundial de la Salud [Internet]. 2013. [citado 2017 feb. 15]

5. Goldman S, Urbano R, Hodapp D. Mortalidad en niños con síndrome de Down [Internet]. 2011, Mar. [citado el 18 de Mar. Del 2017] 118. Disponible desde: https://goo.gl/juSqSv

6. Julio Nazer H., Lucía Cifuentes O. Estudio epidemiológico global del síndrome de Down. Revista chilena de pediatría 2011; 82 (2): 105-112.

7. Consejo Nacional para la Integración de las Personas con Discapacidad. [Internet]. Lima, Perú: CONADIS [citado el 9 Mar. De 2017].

8. Ministerio de Educación. Perú. [Internet]. 2015[citado 16 de Jun. 2017]. Disponible en: https://goo.gl/yhM4gB

9. Tinoco W. El Síndrome de Down y su afectación en el rendimiento escolar enniñosyniñasde7a8años. [Internet]. 2016[citado 16 de Dic. 2017]. Disponible en: http://repositorio.utmachala.edu.ec/bit stream/48000/9652/1/TTUACS\%20D E00005.pdf

10. Arias G. Daniela, Garcia U. Ana. Significado que le dan las madres a la experiencia materna de tener un hijo con síndrome de Down. [Internet]. 2015[citado 2017 Mar 9].

11. Chumo L. Monserrate, Zambrano B. María. El entorno familiar y el desarrollo afectivo de los niños, niñas y adolescentes con síndrome de Down del instituto de educación especial María Buitrón de Zumárraga de la ciudad de Portoviejo, 2014. [Internet]. 2014[citado 2017 Mar 10].

12. Giron Ana. Apego de la madre con un hijo con síndrome de Down que asiste a la fundación Margarita Tejada. [Internet]. 2013. [citado el 2017 Ene 24].

13. Bastidas A. Miriam, Ariza Marriaga Gustavo, Zuliani Arango Liliana. Síndrome de Down: Experiencia maternal de crianza: Entre alegrías y tristezas. Arch Venez Puer Ped [Internet]. 2013. [citado 2017 Mar 12]; 76( 4 ): 151- 158.

14. Ramírez A. Vivencias de los padres en el cuidado del hijo adolescente con Síndrome de Down. [Tesis]. 2016 [citado 05 marzo 2017].

15. Jiménez S, Mamani S. Capacidad De Resiliencia Y Calidad De Vida De Cuidadores De Niños Con Síndrome Down - 
Cebe Auvergne Perú-Francia, Arequipa2015. [Tesis].

16. Chavez R. Sensitividad materna autoreportada en madres de niños con Síndrome de Down. [Tesis].2014 [citado 17 Mar. 2017].

17. Cruz C. Velitt. Bienestar psicológico y actitudes maternas en madres de hijos con necesidades especiales de Trujillo. [Tesis]. 2014[citado el 18 Mar. 2017].

18. Gomez T, Helguero I. Programa "Me relaciono con los demás" para mejorar las habilidades sociales en los niños con Síndrome de Down, en la Institución Educativa $\mathrm{Fe}$ y Alegría $\mathrm{N}^{\circ} 42$, Chimbote2014. [Tesis]. 2014[citado el 12 Dic. 2017]. 